\title{
Color Mathematical Morphology Based on Partial Ordering of Spectra
}

\author{
Marcos Cordeiro d'Ornellas \\ Universidade Federal de Santa Maria (UFSM) \\ Grupo de Processamento de Informação Multimídia (PIGS) \\ Av. Roraima, Prédio 07, Sala 214-216, Campus \\ 97105-900 Santa Maria - RS, Brasil \\ ornellas@inf.ufsm.br \\ José Antônio Trindade Borges da Costa \\ Universidade Federal de Santa Maria (UFSM) \\ Grupo de Processamento de Informação Multimídia (PIGS) \\ Av. Roraima, Prédio 05, Sala 1126, Campus \\ 97105-900 Santa Maria - RS, Brasil \\ jatborgesdacosta@gmail.com
}

\begin{abstract}
Mathematical morphology is based on the principle of ordering. There is no natural way to order colors (being triplets of scalars). A lot of different ordering relations have been proposed in the literature, most on an ad-hoc basis. In this paper, we propose an ordering relation for colors that is based on the natural (i.e. physically plausible) ordering of spectra. Therefore, we ensure that the ordering is independent of the chosen color parameterization. We discuss that part of colorimetric theory that enables us to reconstruct the spectrum given the three color parameters. Furthermore, we present the very basics of the algebraic framework of mathematical morphology. This allows us to embed the presented ordering of colors within the morphological framework such that we can fully exploit the possibilities to define morphological image operators working on color images.
\end{abstract}

\section{Introduction}

Mathematical morphology is grounded on the principle of ordering: ordering of pixel values, ordering of images (being maps from some manifold onto the set of pixel values), and ordering of image operators (being the mappings from images onto images). The algebraic framework for morphology is the complete lattice, where we have a set $V$ of values and a partial ordering relation $\leq$. Given such a simple algebraic structure on the set of pixel values, we are then able to construct most of the morphological tools like erosions, dilations, openings, closings and so on.

Most of the existing research on color morphology field has focused on defining order relationships on a specified color space. Indeed, they map the input color image to the target color space and use an ordering scheme for finding supremum and infimum to compute dilated and eroded images. In [2], color morphological operators have applied on the HSV color space. A lexicographical ordering scheme has been used to find supremum and infimum. This approach has been extended in [3] by defining soft ordering scheme using fuzzy rules.

In [20], the RGB color space is first linearly transformed to the target color space. Then, the transformed colors are ranked using a lexicographic ordering method. The performance of resulted morphological operators in edge detection application has been shown. In [14], the performance of some previously introduced morphological operators (which had used reduced ordering) has been compared and those operators have been mapped to a generic programming framework. Some morphological techniques that had appeared in the literature were reviewed in [21] and a new technique based on vector projections was introduced in that paper. In [22], a reduced ordering approach based on a new geometrical transformation has been introduced and its experimental results for contrast enhancement and edge detection have been reported. In [20], the luminance component in the HLS color space has been used for definition of basic morphological operators. In [15], using color morphological operators defined on the HSI color space for brightness elimination application has been discussed. The use of 
mathematical morphology in the CIE Lab color space has also been discussed in [8]. That approach is based on the use of weighting functions to impose a complete order on a vector space.

Recently, in some studies, color statistical information has been used in color ordering schemes. In [19], morphological operations have been defined based on Mahalanobis distance of colors in the RGB color space. In [12], a majority ordering method has been introduced for color ordering purposes. That approach is based on counting the number of image pixels and ordering the colors accordingly.

From a morphological point of view, defining an ordering relation for colors is all that is required to extend the morphological tools to work with color images. Perhaps the simplest ordering is the partial component-wise ordering of colors. Unfortunately a component-wise partial ordering of colors is dependent on the color model used. A color model is just a way to parameterize a three dimensional color space. As such, the existing parameterization, be it RGB, HLS, XYZ, or CIE Lab, is irrelevant (although it might be extremely handy for particular purposes). Therefore, we aim at an ordering that is independent on the chosen parameterization.

In this paper, we propose an ordering in spectral space, i.e., we define an ordering of spectra (energy distributions over the wavelengths) and through this, induce an ordering on the three dimensional colors. Because the relation of the color model with the spectra that caused the color sensation is explicitly used in our ordering scheme, the ordering is independent of the chosen color representation. The colorimetric theory needed to reconstruct a physical realizable spectrum from the color can be found in section 2. This section on colorimetry is mostly based on the works of [11] and [18]. In section 3 we discuss the algebraic mathematical morphology framework of complete lattices. The standard work on the algebraic theory of morphology is the book of Heijmans [9]. This allows us to open up the entire toolset of mathematical morphology by just defining the basic ingredient: the ordering relation of color pixel values in section 4. Section 5 give some experimental results based on morphological filtering on color images. Conclusions and further research are given in section 6 .

\section{Building a Physical Realizable Spectrum}

Color does not exist objectively as a physical observable in the real world for us to be measured and quantified. Color, as we know it, is a function of the human visual system. The physical phenomena to observe are the energy distribution of electromagnetic waves as a function of wavelength and position. In classical colorimetry, the dependence on wavelength is decoupled from the dependence on position.
Point-wise colorimetry assumes an isolated beam of light falling on the retina with a stationary spectrum $\varepsilon(\lambda)$ (i.e. energy distribution as a function of wavelength). Within the retina, three different color sensitive receptors project the infinite degrees of spectral freedom onto a three dimensional color sensation. The output of the photoreceptors is the weighted integral of the spectral energy distribution. Each of the three different receptors is characterized with sensitivity as a function of the wavelength.

The response $r_{i}$ of each of these receptors to the light spectrum is the weighted integration of the energy distribution $\varepsilon$ and the spectral sensitivity curve $s_{i}$ of that particular receptor ${ }^{1} r_{i}=s_{i}^{T} \varepsilon$. Combining the three sensitivity curves (vectors) in one color matching matrix $M$ we get $c=M^{T} \varepsilon$, where $c=\left(r_{1} r_{2} r_{3}\right)^{T}$ and $M=\left(s_{1} s_{2} s_{3}\right)$.

Human color vision projects an infinite dimensional spectral space $S$ onto a three dimensional color space $C$. Note that the human color space was numerically known long before accurate measurements of the cone sensitivity curves were possible. Using a standard observer, the human visual system is used as a null device to compare the colors resulting from standard primary colors with the colors of monochromatic beams. This results in a color matching matrix that is an affine transformation of the human color matching matrix. The XYZ color matching matrix is the standard colorimetric definition for the human color space. Color space is of rank three as the sensitivity curves are linearly independent and therefore the null space of the projection is of infinite dimension (minus three) as well. Within the infinite dimensional spectral space, the human visual system selects one very specific three dimensional subspace.

In general, it is quite impossible to reconstruct the spectrum $\varepsilon$ that gave rise to the color sensation $c=M^{T} \varepsilon$. It can be approximated as the spectrum $\varepsilon_{F}$ that is within $C$ such that the approximated spectrum results in the same color sensation as the original one $M^{T} \varepsilon_{F}=M^{T} \varepsilon$. The operator $\Pi$ that takes the original spectrum $\varepsilon$ and transforms it into the fundamental spectrum $\varepsilon_{F}$ is the projector that describes the color space $C$ independent of the chosen basis. In colorimetry, this projector is known as Cohen's Matrix [11]. The projector is easily expressed in terms of the color matching matrix $M$.

Consider the spectrum $\varepsilon$ with corresponding color coordinates $c=M^{T} \varepsilon$. The projected spectrum is a linear combination of the columns of the color matching matrix, i.e., there is a vector $c^{\prime}$ such that $\varepsilon_{F}=\Pi_{M} \varepsilon=M c^{\prime}$. The color $M^{T} M c^{\prime}$ corresponding to this spectrum should of course be the same as the original one $M^{T} \varepsilon$. Solving for $c^{\prime}$ (note that

1 we use the common vectorial notation for functions over the wavelength. This makes it easier to apply standard linear algebra in colorimetry. E.g., the inner product $s_{i}^{T} \varepsilon$ should be read as $\int s_{i}(\lambda) \varepsilon(\lambda) d \lambda$. 
$M^{T} M$ is invertible) leads to: $c^{\prime}=\left(M^{T} M\right)^{-1} M^{T} \varepsilon$. Since $M c^{\prime}=\Pi_{M} \varepsilon$ we have $M\left(M^{T} M\right)^{-1} M^{T} \varepsilon=\Pi_{M} \varepsilon$. In addition, since this should be valid for all $\varepsilon$, we obtain:

$$
\Pi_{M}=M\left(M^{T} M\right)^{-1} M^{T} .
$$

Cohen's matrix plays a fundamental role in colorimetry. It allows us to compare color models. Given the sensitivity curves of any tri-stimulus recording device (say the matrix A), then this device is colorimetric if and only if $\Pi_{A}=\Pi_{M}$. The distance between two color models can be expressed in terms of the distance between the associated projector matrices.

The spectrum $\varepsilon$ can be splitted into two parts: its fundamental part $\varepsilon_{F}$ and its black part $\varepsilon_{B}$ such that $\varepsilon=\varepsilon_{F}+\varepsilon_{B}$ and where $\varepsilon_{F}=\Pi_{M} \varepsilon$ and $\varepsilon_{B}=\left(I-\Pi_{M}\right) \varepsilon$. The projector $\left(I-\Pi_{M}\right)$ is the projector on the black space because by construction we have that the black spectrum $\varepsilon_{B}$ does not contribute to the color sensation: $M^{T} \varepsilon_{B}=0$. The black space projector $I-\Pi_{M}$ represents the infinite dimensional (minus three) subspace that is orthogonal to the color subspace.

A second operator that is easily expressed in terms of the color matching matrix is the reconstructor $\Upsilon_{M}$, i.e., the operator that takes a color with respect to some color matching matrix $M$ and results in the canonical spectrum $\hat{\varepsilon}$. It is trivial that this reconstructor is written by:

$$
\Upsilon_{M}=M\left(M^{T} M\right)^{-1} .
$$

Please note that the Grammian Matrix [13] appears in this expression. Only in case the chosen basis is orthonormal, we have that the Grammian $M^{T} M=I$ and thus $\Upsilon_{M}=M$.

Unfortunately, there is no guarantee whatsoever that the reconstructed fundamental spectrum is a physically realizable spectrum: it may have negative values. In many cases, where we are interested in reconstructing the spectrum, the goal is to find a physically realizable spectrum (e.g. to compare spectra for morphological operators or to mimic the color constancy of the human visual system). An algorithm to construct a physically realizable spectrum, given a fundamental spectrum, is elegantly casted into the problem of finding the intersection of two convex bodies [13].

The set of all physically realizable spectra $S_{R}$ is given by $S_{R}=\{\varepsilon \mid \varepsilon \geq 0\}$, i.e., the set of all non-negative spectra. This is obviously a convex set. Now consider the fundamental spectrum $\varepsilon_{F}$ that is not physically realizable, i.e., $\varepsilon_{F} \notin S_{R}$. Of course we may add any black spectrum $b$ from the black space $B$ to $\varepsilon_{F}$ without changing the color. The translated space $\varepsilon_{F}+B$ thus contains only spectra that will lead to the same color sensation as the fundamental spectrum. This space is also convex. Thus, any spectrum in the intersection of $S_{R}$ and $\varepsilon_{F}+B$ is a physically realizable spectrum with the same color sensation as the fundamental spectrum. Finding a spectrum in the intersection is not that hard. Let $\varepsilon^{0}$ be the first estimate. Then, sequentially project it onto the two convex bodies: $\varepsilon^{i+1}=P_{B}\left(P_{R}\left(\varepsilon^{i}\right)\right)$, where $P_{R}$ is the projection on the set of realizable spectra and $P_{B}$ is the projection on the set $\varepsilon_{F}+B$. Iterating this sequence of projections leads to a physically realizable spectrum with the same color sensation as the original fundamental spectrum. A good starting point is, of course, the fundamental spectrum itself.

In practical applications of colorimetry, almost always the XYZ color matching matrix is used to relate RGB color coordinates to the human color space. Given the RGB color coordinates $c_{R G B}$, the coordinates with respect to the XYZaxes $c_{X Y Z}$ are given by the linear transform [17] $c_{X Y Z}=$ $C_{R G B \rightarrow X Y Z} c_{R G B}$, where

$$
C_{R G B \rightarrow X Y Z}=\left(\begin{array}{lll}
0.412 & 0.357 & 0.180 \\
0.213 & 0.715 & 0.072 \\
0.019 & 0.119 & 0.950
\end{array}\right) .
$$

The reconstructor of the fundamental spectrum given an RGB color, thus, is:

$$
\Upsilon=M_{X Y Z}\left(M_{X Y Z}^{T} M_{X Y Z}\right)^{-1} C_{R G B \rightarrow X Y Z} .
$$

The reconstructor operator $\Upsilon$ is illustrated in figure 1 . This figure shows spectra graphic where negative-going RGB values have been clipped to zero. The red/green/blue traces show the (non-clipped, linear-light) RGB device components. Note the negative lobes, particularly of the red channel between the 460 and 550nm part of the simulation. The blue-green part of the spectra does not appear particularly convincing. It is clear from this figure that the basis of fundamental spectra is not physically realizable. In real pictures of natural scenes, highly saturated colors are fortunately rarely found. To make it so, we will use the iterative algorithm discussed above and define the projector $P_{R}$ as the clipping operation:

$$
P_{R}(\varepsilon)_{i}=\left\{\begin{array}{cl}
\varepsilon_{i} & : \varepsilon_{i}>0 \\
0 & : \varepsilon_{i} \leq 0
\end{array}\right.
$$

and the projector on the set $\varepsilon_{F}+B$ as:

$$
P_{B}(\varepsilon)=\left(I-\Pi_{M_{X Y Z}}\right) \varepsilon+\varepsilon_{F}
$$

The basis of the fundamental spectra can be forced to be physically realizable. For a given set of RGB display-primaries, color science yields a $3 x 3$ matrix transform which maps the CIE XYZ color specification into (linear-light) RGB values for the display device. For saturated (near monochromatic) original colors, the mathematically-derived RGB values typically go 


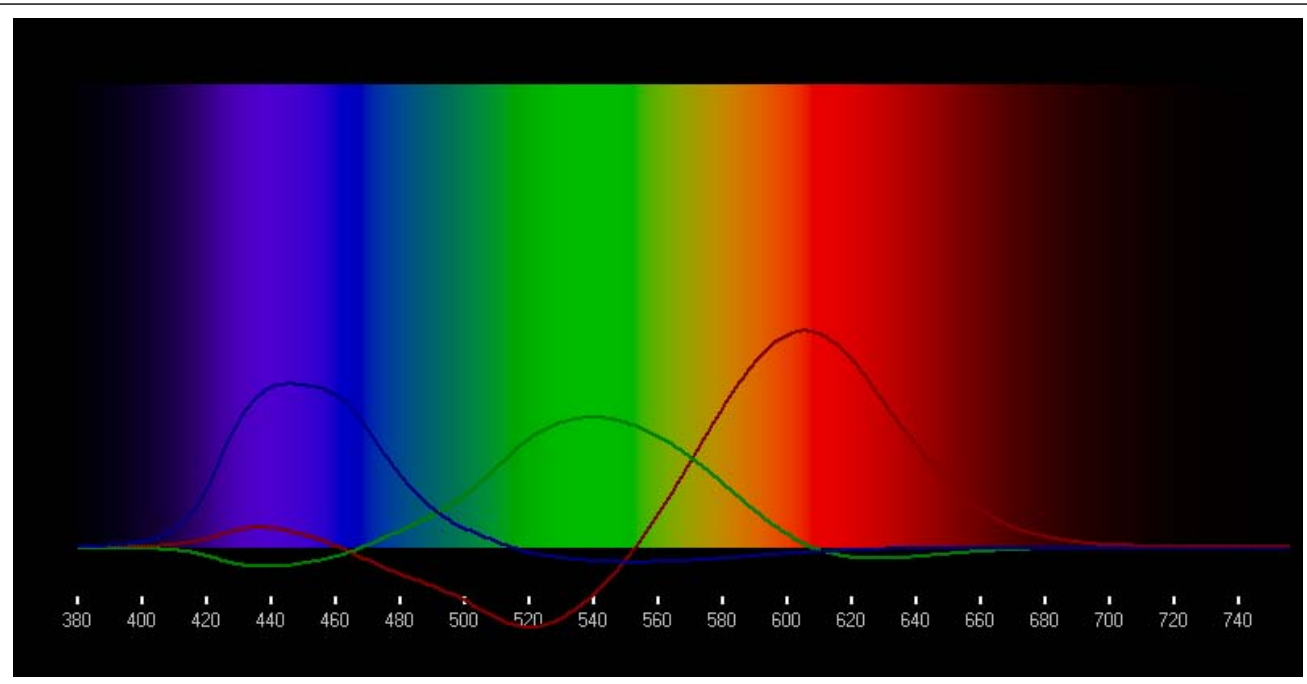

\section{Figure 1. Construction of the fundamental spectrum: The Red, Green, and Blue curves represent the fundamental spectra of the colors $R\left[\begin{array}{lll}1 & 0 & 0\end{array}\right], G\left[\begin{array}{lll}0 & 1 & 0\end{array}\right]$, and $B\left[\begin{array}{lll}0 & 0 & 1\end{array}\right]$ respectively. The fundamental spec- trum of a color $c=[c 1 c 2 c 3]$ is the weighted sum of the depicted spectra.}

negative, because the color is out-of-gamut for the device.

In figure 2 the resulting based is sketched. The three vectors form the columns of a new reconstructor operator $\bar{\Upsilon}$ that maps an RGB color vector to a spectrum that is physically realizable and a metamer of the fundamental spectrum associated with that color. This reconstruction operator will be used a later section to define an ordering on colors induced by an ordering on the reconstructed spectrum.

\section{Bare Bones Mathematical Morphology}

Let $V$ be a set of values and let $E$ be the visual field. In most imaging situations, $E$ is the two dimensional Euclidean plane. An image then is nothing more than a map from $E$ to $V$. The collection of all images will be denoted as $\operatorname{Fun}(E, V)$.

In the abstract theory on mathematical morphology we consider the ordering of pixel values (elements from the value set $V$ ), the ordering of images (elements from $\operatorname{Fun}(E, V)))$ and the ordering of image operators as well. The ordering is formalized with the notion of a complete lattice [5] [6].

The basic building block in a complete lattice is the partial ordering relation $\leq$, defined on a value set $V$. A relation $\leq$ is called a partial ordering in case (i) $v \leq v$ (reflexivity), (ii) $v \leq w$ and $w \leq v$ implies $v=w$ (anti-symmetry) and (iii) $u \leq v$ and $v \leq w$ implies $u \leq w$ (transitivity).

In case a partial ordering relation $\leq$ on the value set $V$ is defined, such that all (even the infinite) subsets of $V$ have a supremum (least upper bound) and an infimum (greatest lower bound $),(V, \leq)$ is a complete lattice. We introduce the notions of the supremum operator $\vee$ and the infimum operator $\wedge$ working on subsets of $V$.

Within a complete lattice, we define erosion and dilation operators ( $\epsilon$ and $\delta$, respectively). An erosion is any operator that distributes over the infimum $\left(\epsilon\left(\wedge_{i} v_{i}\right)=\wedge_{i} \varepsilon\left(v_{i}\right)\right)$ and preserves the lattice supremum. Dilation is any operator that distributes over the supremum and preserves the lattice infimum. For any erosion $\epsilon$ we can find a unique dilation $\delta$ such that $\forall v, w \in V$ :

$$
\delta(w) \leq v \Leftrightarrow w \leq \epsilon(v)
$$

A pair of an erosion and dilation satisfying the above relation is called an adjunction. Given an adjunction $(\epsilon, \delta)$ on a complete lattice the following results can be easily proven [9]:

1. $\epsilon \delta \geq i d$ and $\delta \epsilon \leq i d$ (where $i d$ is the identity operator);

2. $\epsilon \delta \epsilon=\epsilon$ and $\delta \epsilon \delta=\delta$;

3. $\alpha=\epsilon \delta$ is an opening (i.e. $\alpha \leq i d, \alpha^{2}=\alpha$ and $\alpha$ is increasing;

4. $\gamma=\delta \epsilon$ is a closing (i.e. $\gamma \geq i d, \gamma^{2}=\gamma$ and $\gamma$ is increasing.

These results are central in the morphological theory. Because their proof only depends on the existence of a complete lattice and an adjunction, the above properties of erosions and dilations result for free once we have selected 


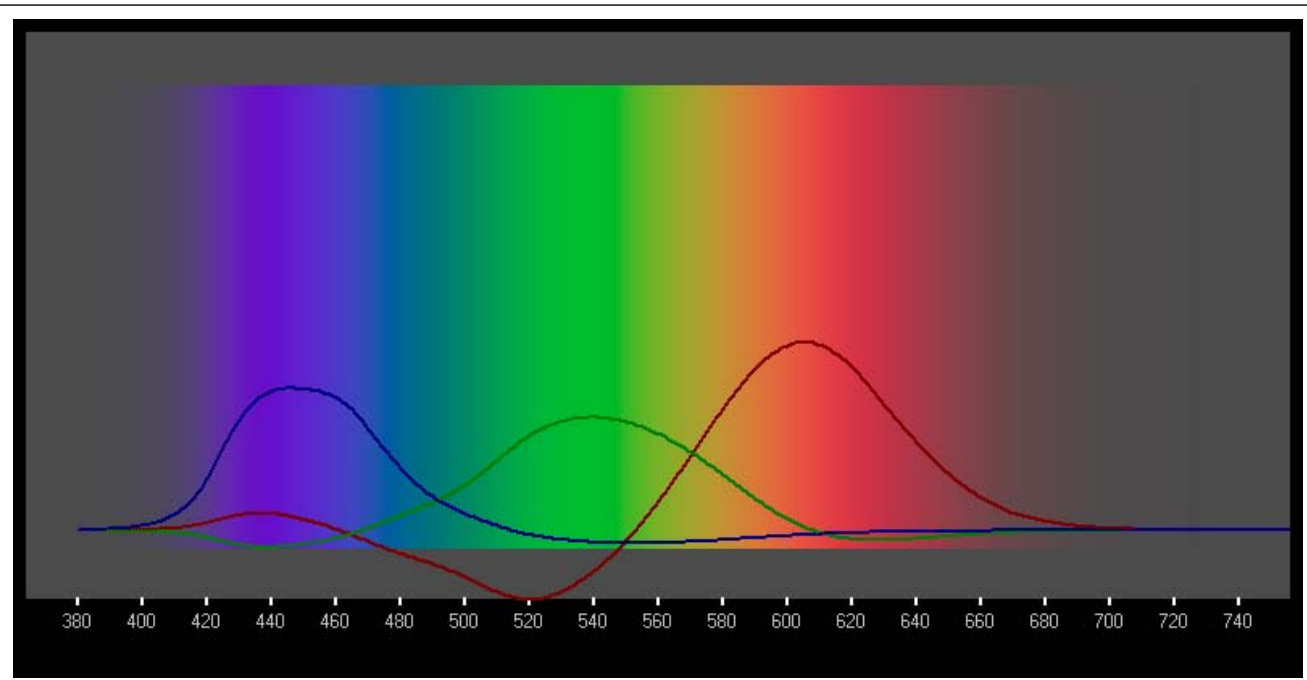

Figure 2. Addition of an uniform amount of white across to the entire spectra, sufficient to lift all but the red component out of the negative. This graphic looks much more believable like we have projected real spectra onto a piece of paper.

a complete lattice an adjoint erosion and dilations for the problem at hand. In the next section we will look at the complete lattice of spectra.

The construction of morphological operators working on images can be seen as a two step process. First look at a complete lattice for the set of pixel values. At this stage we are not considering images at all. The ordering $\leq$ compares pixels values, i.e., elements of $V$. The erosions and dilations defined in the pixel lattice also work on pixel values. A common example of a pixel lattice is the set of real scalars with the usual ordering of the real numbers. Note that there is not just one erosion and dilation pairs in this pixel lattice. In fact, an infinite number of adjunctions can be defined: any pair $\left(\phi, \phi^{-1}\right)$, where $\phi$ is a bijection on $V$, is an adjoint pair of erosion and dilation [10].

Once we have constructed a pixel lattice, we can lift this algebraic structure to the set of images, being mappings from the image domain $E$ to the pixel lattice $V$. Given the partial ordering $\leq$ defined in the pixel lattice, a partial ordering on the set of images is induced. Let $f$ and $g$ be two images (i.e., $f, g \in F u n(E, V)$ ), then we define the ordering:

$$
f \leq g \quad \Leftrightarrow \quad \forall x \in E: f(x) \leq g(x) .
$$

We thus overload the meaning of the ordering symbol $\leq$ to work both on pixel values as well as images, bearing in mind the ordering of images is induced by an ordering of pixel values.

Within the reconstructed image lattice, we can now build erosions and dilations in the image lattice based on erosions and dilations in the pixel lattice. It has been proven in [10] that every erosion $\iota$ in the image lattice can be built as the infimum over erosions $\epsilon$ in the pixel lattice:

$$
(\iota f)(x)=\epsilon_{x, y}(f(y)),
$$

where $\epsilon_{x, y}$ is an erosion for every pair of positions $x$ and $y$. The adjoint dilation on images is equivalently defined as the supremum over the adjoint pixel lattice dilations. Equation 9 will be the starting point in the following section to build erosions and dilations for color images.

\section{Color Morphology}

\subsection{Color Ordering Schemes}

The problem of ordering multivariate data is not limited to the color ordering in mathematical morphology field. In several research fields like multi-objective optimization, multi-sensorial fusion and so forth, the need has been arisen to rank multivariate data. Although there is no natural means for total ordering of multivariate data, much work has been done to define concepts such as median, range, and extremes for multivariate analysis [4]. Barnett [1] has investigated the possible use of incomplete ordering relations for multi-dimensional data and has proposed four possibilities: marginal ordering, reduced ordering, partial ordering, and conditional ordering.

In the marginal ordering scheme, ranking takes place within one (or more) of the marginal sets of samples (i.e., 
scalar ranking is performed for each component). Thus, to order a collection of color vectors using marginal ordering the components in each spectral band are ordered independently. Morphological operations which are defined using marginal ordering are referred to as component-wise operations. Because the component images are filtered separately by using the marginal ordering, there is a possibility of altering the spectral composition of resulted image.

In the reduced ordering scheme, each multivariate observation is reduced to a single value by a transformation function of component values. Then, the multivariate samples are ranked according to this scalar value. In the partial ordering scheme, multivariate samples are grouped into some distinct subsets such that the members of each subset have the same ordering value. In the conditional ordering, multivariate vectors are conditionally ordered based on the value of different components. One type of conditional ordering schemes, the lexicographic ordering, has been used in many studies for color ordering purposes. In this scheme, first, the most significant component is chosen and multivariate ordering is done according to its value. Then, for those vectors having the same ordering level, values of the second most significant components are compared [2] [3].

All of the above mentioned approaches have been derived from scalars defined by distances in the color space. Different approaches can be considered, such as: mapping of initial data vectors (colors) into more familiar objects such as one-dimensional functions, or two dimensional polygons.

\subsection{Finding an Ordering Relation in the Spectral Space}

A color image is a mapping from a spatial domain $E$ to the set of color values. A color value is a three element vector of scalars. It depends on the chosen color model what these scalars represent. A color model is a way to parameterize the three dimensional color space. As such the usual parameterization, being RGB, HLS, XYZ, or CIELab, is irrelevant.

The difficulty in defining morphological operators that work on color images is that an ordering relation for color values is needed. An obvious choice is to extend the scalar ordering to work on three vectors. Let $c$ and $c^{\prime}$ be two colors. Then, we can define a partial ordering on the set of all color values:

$$
c \leq c^{\prime} \Leftrightarrow i=1,2,3: c_{i} \leq c_{i}^{\prime} .
$$

Many authors [20] [21] [7] have already indicated that such a component-wise partial ordering in a vector space is questionable when applied to colors. The main disadvantage is that such an ordering is dependent on the cho- sen color parameterization. Three dimensional color space ows its meaning as a particular and well defined subspace of the infinite dimensional spectral space $(N$-dimensional in the vectorial model). Therefore, any plausible ordering of colors should be related to the orientation of color space in spectral space to make it independent of the irrelevant choice of the basis vectors in spectral space of color (sub)space.

In this section we will first look at an ordering scheme that works on spectra, which are physical entities, largely independent on a choice of representation. It must be noted that order preserving scalings of both the wavelength as well as the energy axes are the only physically plausible change of representation. Later we will use the reconstruction of a physical plausible spectrum from the measured color vector, to induce an ordering in color space. By construction, such an ordering is independent on the chosen color model.

A natural partial ordering in spectral space $S$ is the element-wise ordering. Let $\varepsilon$ and $\varepsilon^{\prime}$ be two spectra, then the ordering relation $\leq$ is defined as:

$$
\varepsilon \leq \varepsilon^{\prime} \quad \Leftrightarrow \quad \forall i: \epsilon_{i} \leq \epsilon_{i}^{\prime}
$$

This partial ordering of spectra is known in colorimetry and is called a dominance hierarchy [11]. The supremum and infimum of spectra is also defined as the element-wise supremum of the spectra and the infimum of the spectra respectively.

In practice, the color is represented in the RGB color model for practical and industrial reasons. Therefore, all we know is the color $c$ and not the spectrum $\varepsilon$. We have already shown in section 2 that it is possible to reconstruct a physical realizable spectrum $\hat{\varepsilon}$ that is in accordance with the observed color $c$ :

$$
\hat{\varepsilon}=\bar{\Upsilon}_{M_{R G B}} c
$$

with $\bar{\Upsilon}_{M_{R G B}}$ the physical realizable reconstruction operator. Based on the natural ordering of spectra, we then define an ordering on colors:

$$
c \leq c^{\prime} \Leftrightarrow \bar{\Upsilon}_{M_{R G B}} c \leq \bar{\Upsilon}_{M_{R G B}} c^{\prime}
$$

It can be proven that the above definition of the relation $\leq$ working on the colors is indeed a partial ordering. The reflexivity and transitivity properties for a partial ordering are easily verified. The anti-symmetry property is only fulfilled in case the projector matrix $\bar{\Upsilon}_{M_{R G B}}$ has linear independent columns, which is indeed the case.

The supremum and infimum operators for colors are easily identified given the above ordering. The supremal color 
$\vee_{i}^{c^{i}}$ of a collection of colors $\left\{c^{i}\right\}$ is the color that corresponds with the supremum of the reconstructed spectra $\vee_{i} \bar{\Upsilon}_{M_{R G B}} c^{i}$. The associated color can be found by projecting the spectrum again. This leads to the following definition of the supremum operator on colors:

$$
\bigvee_{i} c^{i}=M_{R G B}^{T}\left(\bigvee_{i} \bar{\Upsilon}_{M_{R G B}} c^{i}\right)
$$

The infimum operator is defined in a similar fashion.

There are many possible ways to define adjoint pairs of erosions and dilations for color according to the equation 9 . The simples erosion $\epsilon_{x, y}$ is the flat erosion:

$$
\epsilon_{x, y}(c)=\left\{\begin{array}{cc}
c & : x-y \in S \\
\infty & : x-y \notin S
\end{array}\right.
$$

where $S$ is the structuring element defining the local neighborhood(support) of a pixel. With this notion of the erosion of color images, the minimal color in a neighborhood of a pixel is selected (the familiar erosion being the local minimum filter).

A more challenging choice of constructing morphological operations working on color images is to use the idea of first reconstructing the spectrum, then define an erosion in spectral space and, after that, project the spectrum back again to color space. These more challenging erosions have not been implemented yet. A logical candidate would be the one dimensional erosion of the spectrum using an erosion working on one dimensional scalar functions.

\section{Experimental Results}

Experiments have been carried out with morphological filters on a set of color images in order to investigate the results in noise removal. Figure 3 gives subjective examples of a color opening using a flat $3 x 3$ disk, compared to some methods described in the literature. Figure 3(a) represents the original image and figure 3(b) represent the same image, corrupted by a scatter RGB noise, which is used for our comparison purposes. Figure 3(c) is the result obtained by applying the lexicographical approach, described in [3] and [20]. Figure 3(d) and represent the result obtained by applying the solutions described in [21] and [15] respectively. In figure 3(e), the result is obtained by the majority sorting scheme [12]. Finally, figure 3(f) shows the results based on the physically realizable ordering of spectra.

\section{Conclusions and Further Research}

Mathematical morphology image operators working on color images can only be defined based on a partial ordering of colors. As colors are encoded as triplets, an ordering is not so straightforwardly formulated as it is for scalar images. Most of the color orderings proposed in the literature are selected on a rather $a d-h o c$ basis instead of based on first principles.

In this paper we required that the ordering of colors be independent of the model used to represent colors. It seems that such a requirement is the only one compatible with a lack of a priori knowledge: all colors are equal. To that end, we have introduced a partial ordering of colors based on the natural ordering of spectra. Using well-known techniques from colorimetry we have defined an operator that reconstructs a non-negative spectrum, given a color encoded as a RGB triplet. The partial ordering of spectra is the pointwise ordering of functions. In the reconstructed spectral domain, we cam perform erosions and dilations at the pixel level and finally we return to the three component color representation by projecting the spectra resulting from the morphological operations on the RGB color basis.

Such an approach is evidently independent on the chosen color model. It does however require that it is known how to reconstruct the fundamental spectrum from a color. Color calibration is therefore a need in the proposed approach.

Modern algebraic morphology theory provides the framework of what is possible in constructing morphological operators. It does not, however, help us in selecting from several possibilities, the ones that are in accordance with either required symmetries of our visual system or with a priori knowledge of the imaging system. In this paper we have joined the necessary tools both from the field of colorimetry and from the field of mathematical morphology to look at all possibilities to define morphological operators that work on color images in a way that is independent of an arbitrary choice of representation. The result of applying the proposed approach was compared with that of the other morphological openings in the literature and it was shown that our approach give better results.

\section{References}

[1] V. Barnett. The ordering of multivariate data. Journal of Royal Statistical Society A, 3:318-355, 1976.

[2] T. Carron and P. Lambert. Color edge detection using jointly hue, saturation, and intensity. IEEE 1st International Conference on Image Processing (ICIP'94), pages 977-981, 1994.

[3] J. Chanussot and P. Lambert. Total ordering based on space filling curves for multivalued morphology. Proceedings of the International Symposium on Mathematical Morphology (ISMM'98), pages 51-58, 1998.

[4] M. Comer and E. Delp. An empirical study of morphological operators in color image enhancement. Proceedings of the SPIE Conference on Image Processing Algorithms and Techniques III, February 10-13, 1992, San Jose, California, 1657:314-325, 1992. 
[5] J. Crespo and R. W. Schafer. The flat zone approach and color images. In Mathematical Morphology and Its Applications to Image and Signal Processing, Dordrecht, 1995. Kluwer Academic Publishers.

[6] J. Goutsias, H. J. A. M. Heijmans, and K. Sivakumar. Morphological operators for of image sequences. Computer Vision and Image Understanding, 62:326-346, 1995.

[7] C. Gu. Multivalued Morphology and Segmentation-based Coding. PhD thesis, Ecole Polytechnique Federale de Laussane - EPFL, Lausanne, Switzerland, 1995.

[8] A. Hanbury, and J. Serra. Mathematical Morphology in the L*a*b* Colour Space. Technical Report N-36/01/MM, 2001.

[9] H. J. A. M. Heijmans. Morphological Image Operators. Academic press, Boston, 1994.

[10] H. J. A. M. Heijmans and C. Ronse. The algebraic basis of mathematical morphology - part I: Dilations and erosions. Computer Vision, Graphics and Image Processing, 50:245295, 1990.

[11] J. Koenderink and A. Kappers. Color Space. Utrecht University, 4th Edition, 1998.

[12] A. Ledda and W. Philips. Majority Ordering for Colour Mathematical Morphology. Proceedings of the XIIIth European Signal Processing Conference, Antalya, Turkey, 2005.

[13] A. Mertins. Signal Analysis John Wiley and Sons, Ltd, 2001.

[14] M. C. d'Ornellas and R. v.d. Boomgaard. Generic algorithms for morphological image operators - a case study using watersheds. Proceedings of the International Symposium on Mathematical Morphology (ISMM'98), pages 323-330, 1998.

[15] F. Ortiz and F. Torres. Vectorial Morphological Reconstruction for Brightness Elimination in Colour Images. Real-Time Imaging, 10, 2004, pp. 379-387.

[16] C. Ronse. Why mathematical morphology needs complete lattices. Signal Processing, 21:129-154, 1990.

[17] G. Sharma and H. J. Trussel. Digital Color Imaging. IEEE Transactions on Image Processing, 6(7):901-932, July 1997.

[18] D. Travis. The visual system. In Effective Color Displays, London, 1991. Academic Press.

[19] M. T. Ureikat, and H. M. Al-Otum. Angle-Values Mahalanobis-Based Image Morphological Operators. Optical Engineering, Vol. 43, Issue 6, June 2004, pp. 1280-1292.

[20] M. Yu, R. Wang, G. Jiang, X. Liu and T. Y. Choi. New Morphological Operators for Color Image Processing. IEEE Region 10 Conference (TENCON), 2004, pp. 443-446.

[21] M. Wheeler, M. A. Zmuda. Processing Color and Complex Data Using Mathematical Morphology. Proceedings of the IEEE 2000 National Aerospace and Electronics Conference (NAECON), 2000, pp. 618-624.

[22] E. Zaharescu, M. Zamfir and C. Vertan. Color MorphologyLike Operators Based on Color Geometric Shape Characteristics International Symposium on Signals, Circuits and Systems, 2003, pp. 145-148.

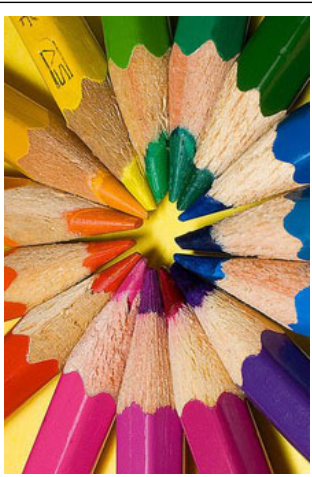

(a)

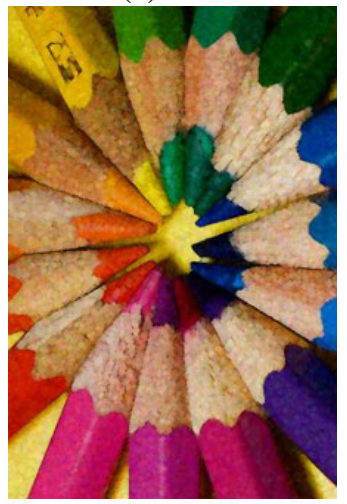

(c)

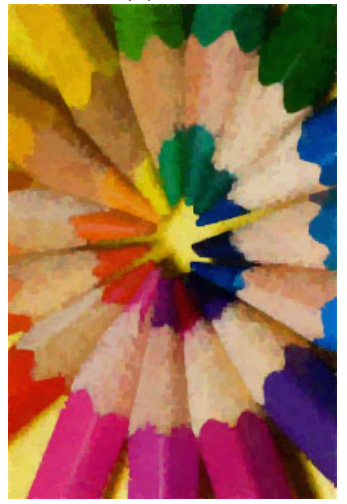

(e)

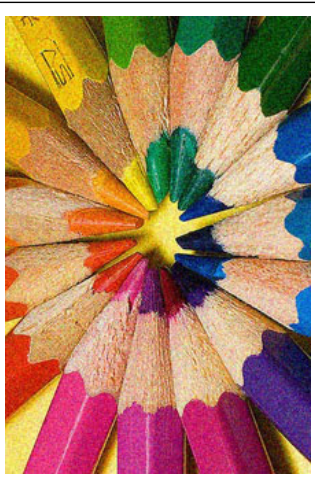

(b)

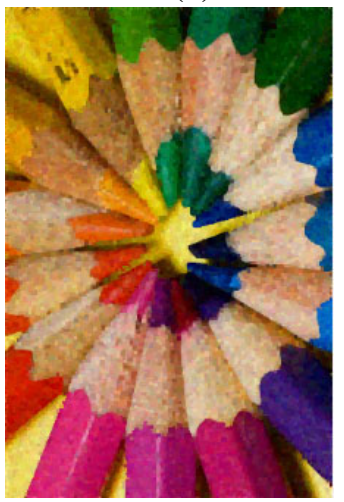

(d)

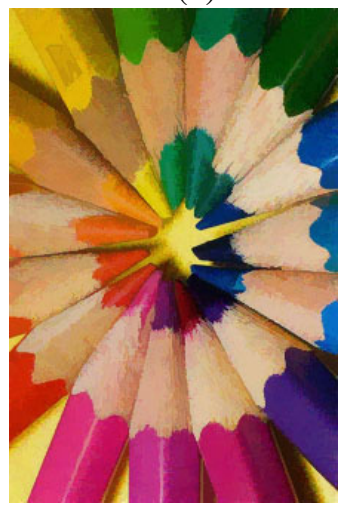

(f)
Figure 3. Illustrative results of applying a color opening based on the methods in the literature and our proposed approach. According to these figures, our proposed method gives better results. 